\title{
Magnetic-field effects on localization in a fractal lattice
}

\author{
X. R. Wang \\ Department of Physics, The Hong Kong University of Science and Technology, Clear Water Bay, Hong Kong
}

(Received 5 July 1995; revised manuscript received 5 January 1996)

\begin{abstract}
Magnetic-field effects on structure-induced localization in a fractal lattice are investigated by studying the usual tight-binding model on a Sierpinski gasket in the presence of a magnetic field. We find that a magnetic field can drastically change properties of states which are localized with high degeneracy in the absence of a magnetic field. In the presence of a magnetic field, the degeneracies of those localized states are broken, and each of them becomes many extended states. Around these new extended state regions, the number of states whose localization length is larger than a certain value decreases with the logarithm of the localization length in a power-law fashion. The exponent of this power-law behavior does not depend on the strength of the magnetic field and the energy range chosen. [S0163-1829(96)03118-9]
\end{abstract}

The energy spectrum and localization of a noninteracting system in a fractal lattice have been the subject of many papers. ${ }^{1-5}$ In a highly correlated space (self-similar) like a fractal, localized eigenstates can exist. ${ }^{1-3}$ However, it is known that the observed localization phenomena on a fractal are fundamentally different from Anderson localization. The usual Anderson localization is due to the incoherent scattering by unorganized scatters (disorders), ${ }^{6}$ and the localization in a fractal space like the Sierpinski gasket is because of the finite ramifications and self-similar lattice structure. The latter is called structure-induced localization.

Domany et $a l .{ }^{1}$ have given a detailed analysis of the electronic structure for the tight-binding model on a Sierpinski gasket. In the absence of a magnetic field, a few extended states exist while the majority of the eigenstates are highly degenerate localized states. The energy spectrum is a Cantor set. States are localized because renormalized hopping coefficients are exactly zero due to the special lattice structure of the Sierpinski gasket. Banavar, Kadanoff, and Pruisken ${ }^{5}$ generalized Domany et al.'s approach to study the energy spectrum of a Sierpinski gasket lattice in the presence of a magnetic field. They found that the introduction of a phase (due to the magnetic field) breaks all the degeneracies which exist in and dominate the zero-field solution. From an escape-rate type of argument, they concluded that the energy spectrum is still isolated with zero measure though it is considerably broader than that in the absence of a field. The results from direct diagonalization and Green's-function calculations are consistent with the majority of the eigenstates being truly extended despite the finite order of ramification of the fractal lattice. Very recently, ${ }^{4}$ this author has proposed an alternative method to study localization. Instead of solving a Schrödinger equation, it is enough to study the asymptotic behavior of effective hopping coefficients if one wants to calculate the localization length only. The asymptotic behavior of the effective hopping coefficient is given by its recursion relation. The spirit of this method had also been used to study other quantities. ${ }^{7}$ For a finitely ramified fractal lattice like the Sierpinski gasket, the exact recursion relation can be obtained. For the tight-binding model on a Sierpinski gasket in the absence of a magnetic field, one can show that those extended states obtained by Domany et al. are generated from one of the fixed points of the recursion relation, and the localization length of gap states reveals a self-similar structure. 4

In this paper, I will generalize the approach of Ref. 4 to a noninteracting system on the Sierpinski gasket in the presence of a magnetic field. The usual tight-binding model, with only the nearest-neighbor hopping, is used to describe the noninteracting system. The Hamiltonian is

$$
\mathscr{H}=\sum_{\langle i j\rangle} t e^{i \phi_{i j}} c_{i}^{\dagger} c_{j}
$$

In Eq. (1), $\langle i j\rangle$ denote the nearest-neighbor site pairs on the Sierpinski gasket, $\phi_{i j}$ is the phase experienced by the hopping coefficient and bond $i j$ due to a magnetic field, $c_{i}^{\dagger}$ and $c_{i}$ are the usual creation and annihilation operators, respectively, and $t$ is the magnitude of the hopping coefficient. The magnitude of the hopping coefficient $t$ is assumed to be the same. The magnetic field is introduced through the "Peierls ansatz," 8 namely, the only effect of a magnetic field on a tight-binding model is to add a phase to the hopping coefficient. Following Banavar, Kadanoff, and Pruisken, ${ }^{5}$ I will study a problem in a special magnetic field in which the magnetic flux in an elementary upward-pointing triangle, labeled $U$ in Fig. 1(a), is equal to $\Phi=3 \phi$, and the magnetic flux in an elementary downward-pointing triangle, labeled $D$ in Fig. 1(a), is equal to $\Phi=-3 \phi$. I will use a gauge in which all bonds in the direction of the arrows in Fig. 1(a) have a hopping coefficient $t e^{i \phi}$, and all bonds opposite to the arrows have a hopping coefficient $t e^{-i \phi}$. This field is nontrivial, and I choose it for convenience. Other types of fields could have been chosen, but the qualitative results obtained in this paper should not change. Obviously, there is no disorder in the system. I will show that the nature of those highly degenerate localized states found by Domany et al. ${ }^{1}$ will change dramatically. It can be explicitly shown that each of those highly degenerate localized states becomes many extended states. In order to find out whether bands of extended states exist in the presence of a magnetic field, I will carry out a "counting" experiment. I find that, around these new extended-state regions, the number of states whose localization length is larger than a certain value decreases with 

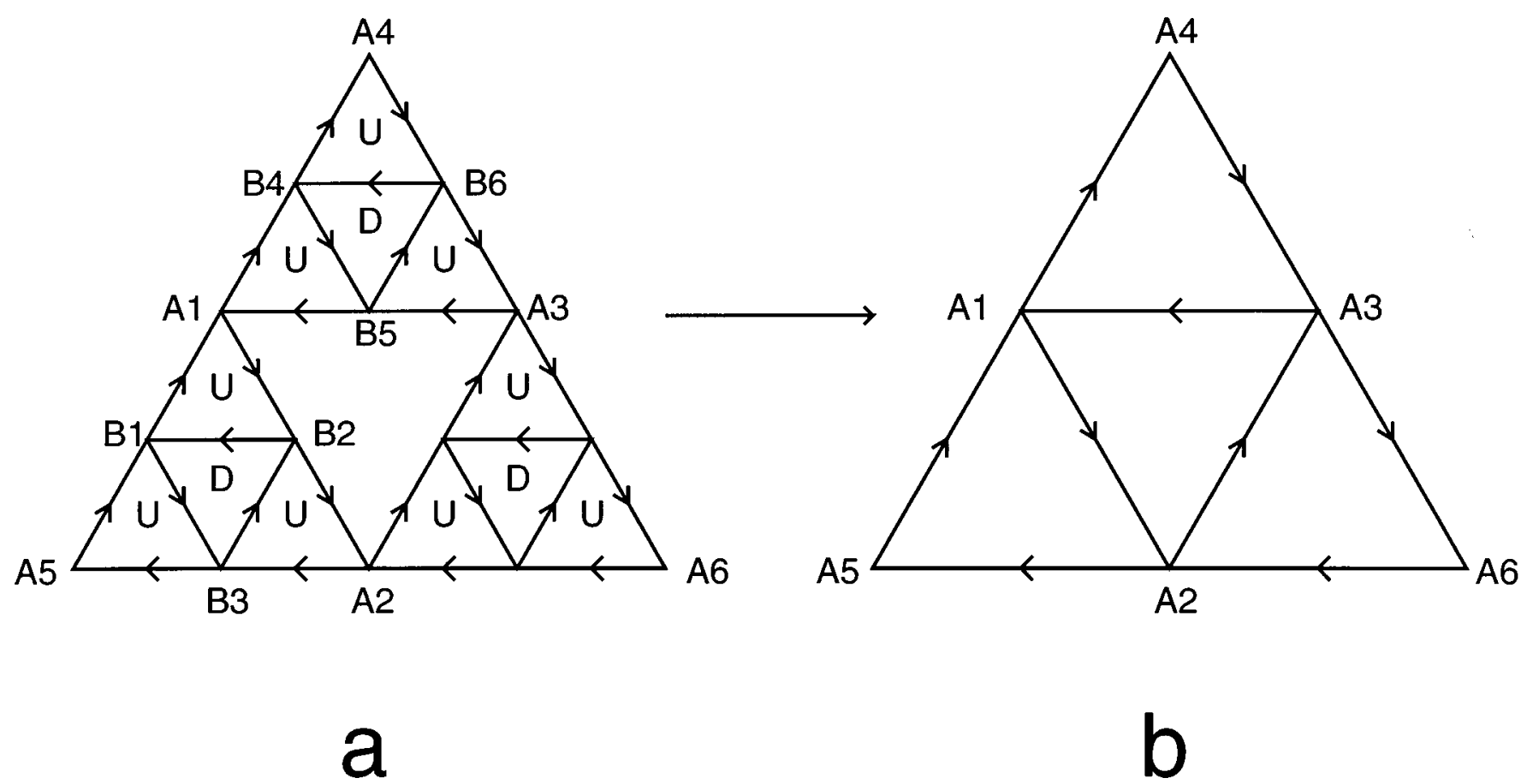

FIG. 1. The Sierpinski gaskets before and after decimation. The $A$ 's and $B$ 's are the wave-function amplitudes at the corresponding sites. The magnetic flux is $3 \phi$ in an upward-pointing triangle labeled $U$, and $-3 \phi$ in a downward-pointing triangle labeled $D$.

the logarithm of the localization length in a power-law fashion. The exponents of this power-law behavior around different energy regions are the same and they do not depend on the magnetic field. This power-law behavior implies that all extended states are isolated from each other. These results are consistent with those of Banavar, Kadanoff, and Pruisken. $^{5}$

Let the eigenstate, the eigenvalue $\epsilon$, be $|n\rangle=\Sigma_{i} A_{i} c_{i}^{\dagger}|0\rangle$, where $|0\rangle$ is the vacuum state, and $A_{i}$ is the amplitude of the wave function $|n\rangle$ at site $i$. I am interested in the nature of the wave function, i.e., how $A_{i}$ behaves as $i \rightarrow \infty$. In my previous publication, ${ }^{4}$ I have shown that the localization length can be obtained from the asymptotic behavior of the effective hopping coefficient $t(R)$. For a localized state of localization length $\xi$, the effective hopping coefficient between two sites of a large distance $R$ behaves as $t(R) \sim e^{-R / \xi}$ while $t(R)$ will not go to zero as $R \rightarrow \infty$ for an extended state. In the absence of a magnetic field, i.e., $\phi_{i j}=0$, I found the following recursion relation for $t$ by using the decimation method: ${ }^{4}$

$$
t_{1}= \begin{cases}\frac{t^{2}}{\epsilon-3 t}, & t \neq-\frac{\epsilon}{2} \\ 0, & t=-\frac{\epsilon}{2}\end{cases}
$$

In the previous publication, ${ }^{4} \mathrm{I}$ demonstrated that two fixed points of Eq. (2), $t^{*}=0$ and $\epsilon / 4$, determine the nature of the wave functions. The fixed point $t^{*}=\epsilon / 4$ characterizes the behavior of extended states, and $t^{*}=0$ that of localized states. Combined with Domany et al.' ${ }^{1}$ work, we know that all allowed extended states are generated from fixed point $t^{*}=\epsilon / 4$, and all allowed localized states are generated back- ward from Eq. (2) by the special point $t=-\epsilon / 2$. The allowed localized states, ${ }^{1}$ therefore are $\epsilon=-2 t, t,(3 \pm \sqrt{13}) / 2, \ldots$. Domany et al. ${ }^{1}$ showed that these localized states are highly degenerate. Special point $t=-\epsilon / 2$, or a state of energy $\epsilon=-2 t$, has a zero effective hopping coefficient between two next-nearest-neighbor sites. The zero effective hopping coefficients are due to the special symmetry and the lattice structure of the Sierpinski gasket. In other words, this is structure-induced rather than disorder-induced localization. These localized states should not be stable against any perturbation. Therefore, in the presence of a magnetic field, they are expected to change dramatically. In order to study quantitatively the change of the localized states, I will use the decimation renormalization method introduced in my previous publication. ${ }^{4}$

In the presence of a magnetic field, one more parameter $\phi$, the magnetic flux (or the phase of the hopping coefficient), should be introduced in addition to the magnitude of the hopping coefficient $t$. It is easy to show that a wave function of energy $\epsilon$ satisfies the following equations:

$$
\begin{aligned}
& \epsilon A_{1}=t\left(B_{1} e^{i \phi}+B_{2} e^{-i \phi}+B_{4} e^{-i \phi}+B_{5} e^{i \phi}\right), \\
& \epsilon B_{1}=t\left(B_{2} e^{i \phi}+B_{3} e^{-i \phi}+A_{1} e^{-i \phi}+A_{5} e^{i \phi}\right), \\
& \epsilon B_{2}=t\left(B_{1} e^{-i \phi}+B_{3} e^{i \phi}+A_{1} e^{i \phi}+A_{2} e^{-i \phi}\right), \\
& \epsilon B_{3}=t\left(B_{1} e^{i \phi}+B_{2} e^{-i \phi}+A_{2} e^{i \phi}+A_{5} e^{-i \phi}\right), \\
& \epsilon B_{4}=t\left(B_{5} e^{-i \phi}+B_{6} e^{i \phi}+A_{1} e^{i \phi}+A_{4} e^{-i \phi}\right), \\
& \epsilon B_{5}=t\left(B_{4} e^{i \phi}+B_{6} e^{-i \phi}+A_{1} e^{-i \phi}+A_{3} e^{i \phi}\right), \\
& \epsilon B_{6}=t\left(B_{4} e^{-i \phi}+B_{5} e^{i \phi}+A_{3} e^{-i \phi}+A_{4} e^{i \phi}\right),
\end{aligned}
$$


where the $A$ 's and $B$ 's are the wave-function amplitudes at the sites shown in Fig. 1(a). Eliminating the $B$ 's in Eq. (3), we can derive an equation which contains only $A$ 's. After some simple algebra, we have

$$
\epsilon A_{1}=t^{\prime}\left(A_{2} \epsilon^{-i \phi^{\prime}}+A_{3} e^{i \phi^{\prime}}+A_{4} e^{-i \phi^{\prime}}+A_{5} e^{i \phi^{\prime}}\right)
$$

where

$$
\begin{gathered}
t^{\prime}=\frac{t^{2} \sqrt{\epsilon^{4}+6 \epsilon^{3} t \cos 3 \phi+11 \epsilon^{2} t^{2}+2 \epsilon^{2} t^{2} \cos 6 \phi+12 \epsilon t^{3} \cos 3 \phi+2 t^{4}+2 t^{4} \cos 6 \phi}}{\epsilon^{3}-7 \epsilon t^{2}-6 t^{3} \cos 3 \phi} \\
\phi^{\prime}=-\phi+\delta, \\
\delta=\left[\operatorname{sgn}\left(\epsilon^{2} \cos 3 \phi+3 \epsilon t+2 t^{2} \cos 3 \phi\right)-1\right] \frac{\pi}{2}+t g^{-1} \frac{\epsilon^{2} \sin 3 \phi}{\epsilon^{2} \cos 3 \phi+3 \epsilon t+2 t^{2} \cos 3 \phi},
\end{gathered}
$$

with $\operatorname{sgn}(x)$ defined as

$$
\operatorname{sgn}(x)= \begin{cases}1 & \text { if } x \geqslant 0, \\ -1 & \text { otherwise. }\end{cases}
$$

In deriving the equation for $\delta$, I have used the fact that one can add an arbitrary integer number of $2 \pi$ 's to $\delta$. The first term in the right-hand side of the equation comes from the fact that $\operatorname{tg}^{-1}(x)$ can only take values in $[-\pi / 2, \pi / 2]$. Obviously, the relevant parameters are $t / \epsilon$ and $\phi$. Therefore, after replacing $t / \epsilon$ by $t$, we have the following recursion relations:

$$
\begin{gathered}
t_{n+1}=\frac{t_{n}^{2} \sqrt{1+6 t_{n} \cos 3 \phi_{n}+11 t_{n}^{2}+2 t_{n}^{2} \cos 6 \phi_{n}+12 t_{n}^{3} \cos 3 \phi_{n}+2 t_{n}^{4}+2 t_{n}^{4} \cos 6 \phi_{n}}}{1-7 t_{n}^{2}-6 t_{n}^{3} \cos 3 \phi_{n}} \\
\phi_{n+1}=-\phi_{n}+\delta, \\
\delta=\left[\operatorname{sgn}\left(\cos 3 \phi_{n}+3 t_{n}+2 t_{n}^{2} \cos 3 \phi_{n}\right)-1\right] \frac{\pi}{2}+t^{-1} \frac{\sin 3 \phi_{n}}{\cos 3 \phi_{n}+3 t_{n}+2 t_{n}^{2} \cos 3 \phi_{n}} .
\end{gathered}
$$

$t_{n}$ is the effective hopping coefficient between two sites of distance $L\left(=2^{n}\right)$, and $\phi_{n}$ is the renormalized phase after decimating $n$ times. It is easy to see that the recursion relations become trivial when $n_{n}=0$, namely, $t_{n+1}=0$ and $\phi_{n+1}=2 \phi_{n}$. The physical explanation of this result is simple. In one decimation, three upward-pointing triangles and one downward-pointing triangle are renormalized to one upward-pointing triangle (Fig. 1). Therefore the total flux in one renormalized upward-pointing triangle is twice that in one upward-pointing triangle before the decimation.

As the fixed point $t^{*}=\epsilon / 4$ plays an important role in giving all allowed extended states in the case of no magnetic field, the fixed point(s) given by

$$
\begin{aligned}
& 1-15 t^{2}-18 t^{3} \cos 3 \phi+36 t^{4}+4 t^{4} \cos ^{2} 3 \phi+72 t^{5} \cos 3 \phi \\
& +32 t^{6} \cos ^{2} 3 \phi=0 \\
& 2 \phi-\left[\operatorname{sgn}\left(\cos 3 \phi+3 t+2 t^{2} \cos 3 \phi\right)-1\right] \frac{\pi}{2} \\
& +\operatorname{tg}^{-1} \frac{\sin 3 \phi}{\cos 3 \phi+3 t+2 t^{2} \cos 3 \phi}=0
\end{aligned}
$$

should also play an important role in the presence of a magnetic field. Unfortunately, recursion relation (5) cannot be solved exactly. However, it is easy to evaluate $t_{n}$ numeri- cally. As mentioned in Ref. 4, we can calculate the localization length $\xi$ of a given energy state by fitting $t_{n}$ to $\exp \left(-2^{n} / \xi\right)$ for large $n$. Figures $2(\mathrm{a})-2(\mathrm{c})$ are the plots of the inverse of the localization length vs the energy for different $\phi$ 's, $\phi=0,0.02,0.04$, in the energy range $[-4 t, 4 t]$. The energy range $[-4 t, 4 t]$ is chosen because the energies of all allowed states must be in the range. ${ }^{1,4}$ It is interesting to see that many extended states appear around $\epsilon=-2 t, t,(3 \pm \sqrt{13}) t / 2, \ldots$ in the magnetic field. In the absence of a magnetic field, they are exactly those allowed highly degenerate localized states found by Domany et al. ${ }^{1}$ This means, as expected, that the degeneracies of these localized states are broken by a magnetic field. However, it is not trivial that they should become extended. As the strength of the magnetic field increases, the induced extended states spread wider and wider. From Figs. 2(a)-2(c), it is not easy to tell whether energy bands are formed around each of those allowed localized states. It is easy to derive from Domany et al.'s ${ }^{1}$ work that the wave functions of all the allowed localized states are separated from each other. The centers of these wave functions also form a Sierpinski gasket. Thus they are very much as if there is one atomic wave function on each of the lattice atoms in a crystal. It is textbook knowledge that, when atoms are brought together to form a crystal, the degeneracy of each atomic level will be broken, and each atomic level is split into many different states due to the 

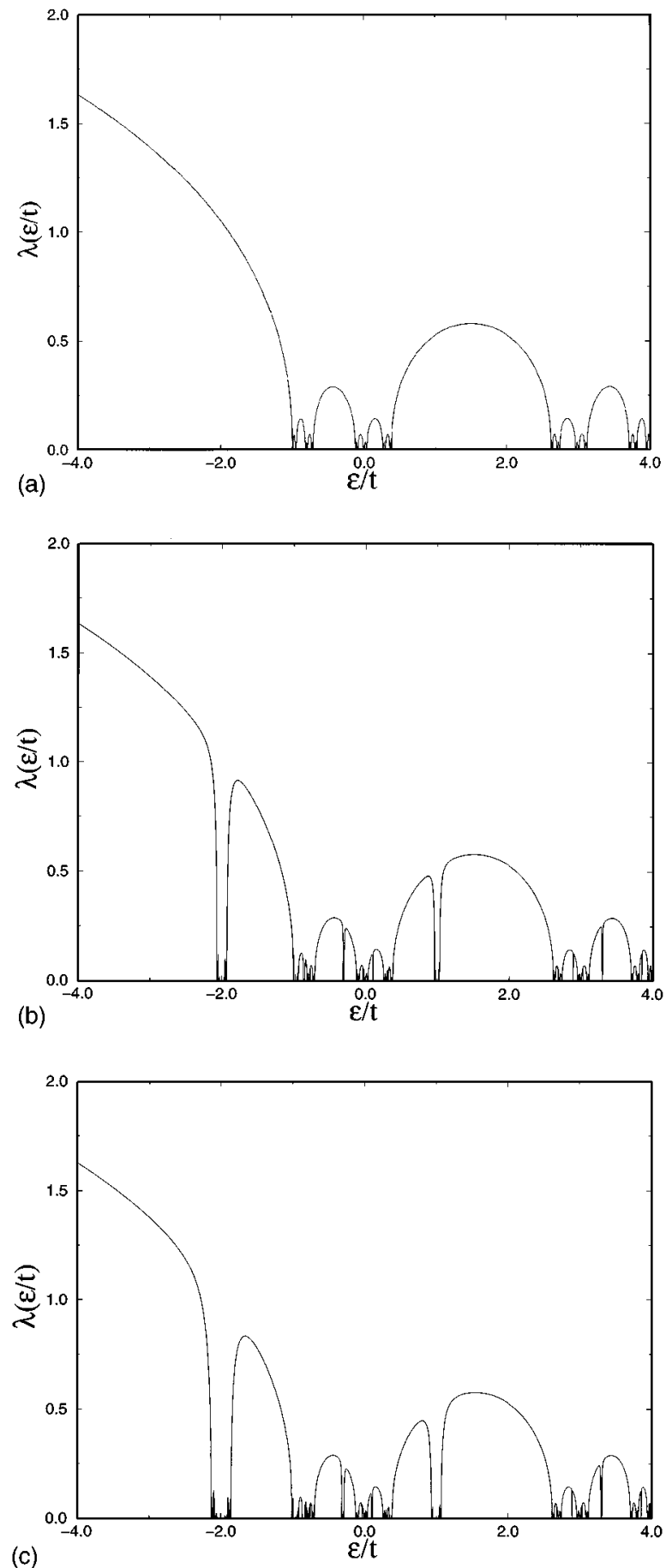

FIG. 2. $\lambda(\epsilon)$, the inverse of the localization length, vs $\epsilon$ for different magnetic fields. (a) $\phi=0$, (b) $\phi=0.02$, and (c) $\phi=0.04$. Those states with $\lambda=0$ are extended.

overlaps of the wave functions on different atoms. The splits of the atomic levels lead to formation of the energy bands in a crystal. In the present case, a magnetic field can be regarded as a way to couple together localized wave functions on different sites. Naively, one may also expect that energy bands are formed when each localized state splits into many different states in a magnetic field. However, Figs. 2(b) and 2(c) show clearly that there are some internal structures about these newly created extended states. In order to address the issue of nonexistence of energy bands in a magnetic
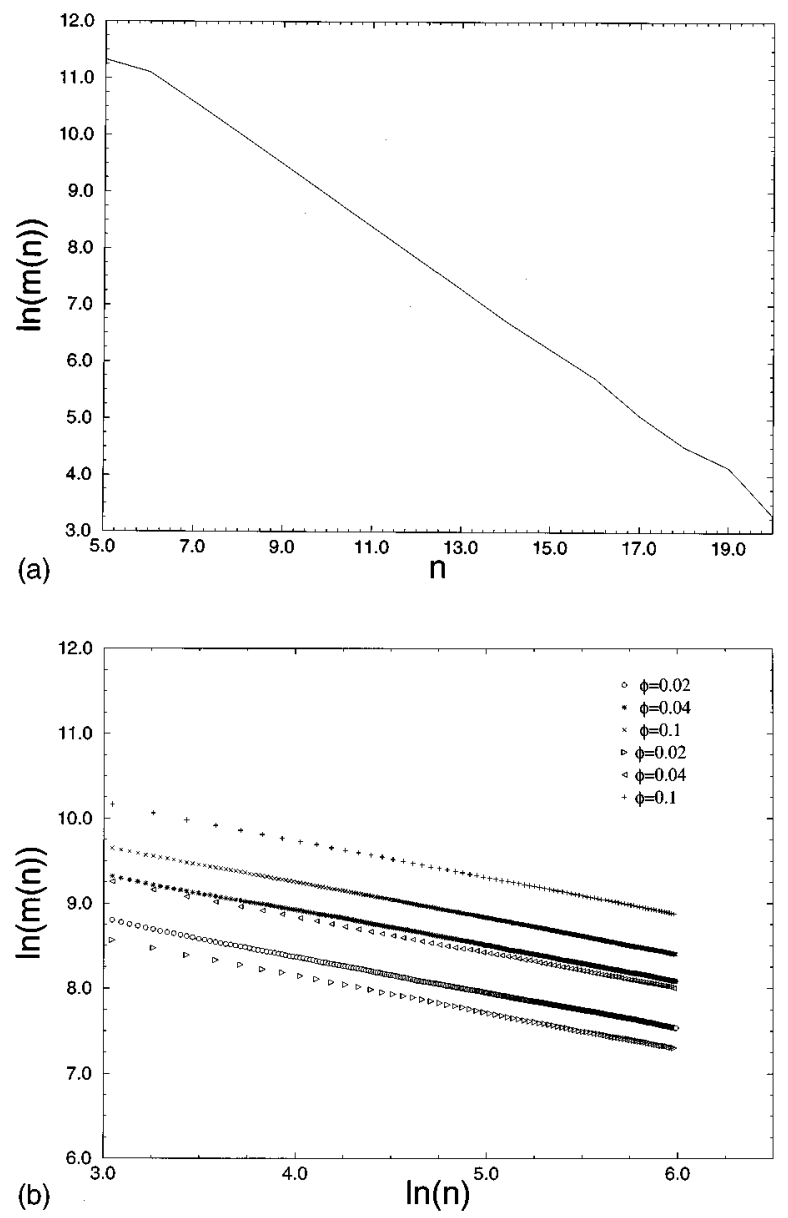

FIG. 3. (a) $\ln m(n)$ vs $n$ in energy range $\epsilon \in[-4 t, 4 t]$ in the absence of a magnetic field. (b) $\ln m(n)$ vs $\ln n$ in the presence of a magnetic field. Open circles, stars, and crosses are for $\phi=0.02,0.04,0.1$, respectively, in energy range $\epsilon$ $\in[-2.4 t,-1.6 t]$. Right triangles, left triangles, and pluses are for $\phi=0.02,0.04,0.1$, respectively, in energy range $\epsilon \in[0.6 t, 1.4 t]$.

field, I carry out a "counting', experiment similar to the "escape-rate", experiment by Banavar, Kadanoff, and Pruisken. ${ }^{5}$ Starting from 80000 equally spaced points in an energy range, say $\epsilon \in[-4 t, 4 t]$, and at a given magnetic field $\phi$, I carry out $n$-step calculations of recursion relation (5) for each point. Then I fit the results by $t_{n} \sim \exp \left(-2^{n} / \xi\right)$ to calculate $\xi(\epsilon)$ for a given $\epsilon$. The localization length is larger than $2^{n}$ if $\xi(\epsilon)$ is numerically zero after $n$ decimations. Let $m(n)$ denote the number of states (out of 80000 ) whose localization length is larger than $2^{n}$. Then $m(n)$ will saturate to a nonzero constant if bands of extended states exist. On the other hand, if at most isolated extended states exist in the energy range, $m(n)$ will approach zero as $n$ increases because the measure of isolated extended states is zero. Figure $3(\mathrm{a})$ is the plot of $\ln [m(n)]$ vs $n$ in the energy range $[-4 t, 4 t]$ in the absence of a magnetic field. Figure $3(\mathrm{~b})$ is the plot of $\ln [m(n)]$ vs $\ln n$ in the presence of a magnetic field $\phi=0.02,0.04,0.1$ in two different energy regions where allowed localized states exist when a field is absent. In the absence of a magnetic field, $m(n)$ decays exponentially with $n$, i.e., $m(n) \sim e^{-\beta n}$ or $m(\xi) \sim \xi^{-\beta^{\prime}}$. In the presence of a magnetic field, however, $m(n)$ decays to zero in power-law 
fashion, $m(n) \sim n^{-\alpha}$, or $m(\xi) \sim(\ln \xi)^{-\alpha^{\prime}}$. In Fig. 3(b), the open circles, stars, and crosses are the numerical results of $\ln [m(n)]$ in the energy region $[-2.4 t,-1.6 t]$ with $\phi=0.02,0.04,0.1$, respectively. The right triangles, left triangles, and pluses are those in the energy region $[0.6 t, 1.4 t]$ with $\phi=0.02,0.04,0.1$, respectively. All of them can be fitted well by parallel lines with $\alpha \simeq 0.43$. Numerical studies in other energy regions with different magnetic fields confirm that the exponent $\alpha$ does not depend on the strength of the magnetic field and energy range chosen. The results imply that the extended states in the presence of a magnetic field are still isolated. However, $m(n)$ has changed from exponential decay to power-law decay. The power-law decay may mean that extended states are highly dense.

In conclusion, we have studied the magnetic-field effects on localization in the Sierpinski gasket lattice. We find that a magnetic field not only can break the degeneracies of a localized state, but also changes this state from a localized to extended. This particular example shows that structureinduced localization is very different from the usual Anderson localization. This difference can be understood from the origins of the two kinds of localization. Anderson localization is due to unorganized incoherent scatterings. There are several features about it. (i) Localized states form a continuous spectrum. When there exists a mobility edge, the energy spectra on both sides of the edge are continued (hydrogenlike localized states in a doped semiconductor are isolated). (ii) Anderson localization is usually stable against small perturbations in the sense that the mobility edge changes continuously with external perturbation. On the other hand, states are localized in a fractal space through coherent destructive interference due to the special symmetry and structure of the space. The localized states usually form a singular spectrum (a Cantor set, for example). Therefore the localization cannot be stable against a small perturbation which changes the symmetry of the system. A magnetic field breaks the lattice symmetry on the Sierpinski gasket. It, then, can destroy the coherent destructive interference similarly to a field suppressing backward scatterings in a disordered system. Thus the degenerate localized states are split into many extended states. This result is interesting and contrary to the common wisdom because a magnetic field usually increases the degrees of irregularity of a pure system. Therefore it usually makes a state more localized in a conventional pure system. ${ }^{9}$ A famous example of such systems may be a particle in a two-dimensional Euclidean lattice without any disorder. Without a magnetic field, all allowed states are extended following the Bloch theorem, but in the presence of an incommensurate magnetic flux many states become localized. ${ }^{10}$ However, the current example shows that wave functions behave differently in a magnetic field, namely, some localized states become extended in a magnetic field. If the above explanation is correct, then we should expect that not only a magnetic field can induce extended states, but also other types of perturbations, such as a small number of lattice defects or disorder, can change some localized states into extended states. ${ }^{11}$ My "counting'" experiment shows that no energy bands form in the presence of a magnetic field. However, I find that the number of states whose localization length is larger than a certain value decreases with the logarithm of the localization length in a power-law fashion. The exponent of this power-law behavior does not depend on the strength of the magnetic field and the energy range chosen.

The author thanks Professor E. Domany, Professor N. Kumar, Dr. Z. Q. Zhang, and Professor Y. Shapir for stimulating discussions. This work was supported by UPGC, Hong Kong, through RGC and RTG grants.
${ }^{1}$ E. Domany, S. Alexander, D. Bensimon, and L. P. Kadanoff, Phys. Rev. B 28, 3110 (1983).

${ }^{2}$ R. Rammal and G. Toulous, Phys. Rev. Lett. 49, 1194 (1982); J. Phys. (Paris) Lett. 44, L13 (1983); R. Rammal, J. Phys. (Paris) 45, 191 (1984); Phys. Rev. B 28, 4871 (1983).

${ }^{3}$ S. Alexander, Phys. Rev. B 27, 1541 (1983); W. Schwalm, M. Schwalm, and K. Rada, ibid. 44, 382 (1991).

${ }^{4}$ X. R. Wang, Phys. Rev. B 51, 9310 (1995).

${ }^{5}$ J. R. Banavar, L. Kadanoff, and A. M. M. Pruisken, Phys. Rev. B 31, 1388 (1985).

${ }^{6}$ P. W. Anderson, Phys. Rev. 109, 1492 (1958).
${ }^{7}$ S. Havlin and D. Ben-Avraham, Adv. Phys. 36, 695 (1987).

${ }^{8}$ E. Medina, M. Kardar, Y. Shapir, and X. R. Wang, Phys. Rev. Lett. 64, 1816 (1990); Y. Shapir and X. R. Wang, Mod. Phys. Lett. B 4, 1301 (1990).

${ }^{9}$ In a disordered system, a magnetic field can also make a state less localized by suppressing the backward scattering. It is well known that a magnetic field can even create extended states in a quantum Hall system through this mechanism.

${ }^{10}$ D. R. Hofstadter, Phys. Rev. B 14, 2239 (1976); M. Kohmoto, L. P. Kadanoff, and C. Tang, Phys. Rev. Lett. 50, 1870 (1983).

${ }^{11}$ N. Kumar (private communication). 